Bei Durchsicht dieser Aufstellung und bei Vergleich der bei den verschiedenen Prüfungsbestimmungen erhaltenen Werte ist Rücksicht darauf zu nehmen, daß die Zahlen in der Spalte $12 \mathrm{zu}$ einem großen Teil nur dadurch erhalten worden sind, daß fehlende Angaben von mir ergänzt wurden. (Siehe die Durchführung und Kritik der betreffenden Prüfung.) Eine endgültige Entscheidung kann auf Grund der in dieser Spalte stehenden Werte allein nicht gefällt werden.

Aus dem Institut fur Pharmakologie und physiologische Chemie der Universität zu Rostock.

\title{
Ueber Ester aromatischer Arsenverbindungen (der p-Benzarsinsäure) mit Aminosäuren und höheren Alkoholen.
}

Von Ernst Sieb urg.

(Eingegangen den 7. III. 1916.)

In einer früheren Arbeiti) wurde die Frage diskutiert, ob es sich bei der Wirkung von Arsenpräparaten auf den Organismus um eine katalytische Hemmung lebenswichtiger Prozesse handelt, oder ob die Wirkung auf chemischer Bindung irgend eines für das Zelleben notwendigen Minimumstoffes des Protoplasmas beruht. Die Ansicht der ,Verankerung“" gewisser Arsenikalien mit dem Zellprotoplasma wurde vornehmlich von $\mathrm{Paul} \mathrm{Ehrlich}$ vertreten. Die schon früher besonders von französischen Autoren verfochtene Annahme, daß es sich hierbei um die Bildung von Arsenlecithinen oder Arsennucleiden handelt, ist noch recht problematisch. Arsenpräparate mit genuinem Eiweiß, die therapeutischen Zwecken dienen sollen, wie z. B. das durch Einwirkung von Arsentrichlorid in Essigsäureanhydrid gewonnene Präparat ${ }^{2}$ ), oder die durch Einwirkung von Arsentrichlorid in alkoholischer Lösung auf Weizeneiweiß dargestellte Substanz ${ }^{3}$ ), dürften kaum als chemisch

1) E. S i e b u r g, Zur Biologie aromatischer Arsenverbindungen, Ztschr. f. physiol. Chem. 97, Heft 2/3, 1916.

2) D. R. P. 201370.

a) D. R. P. 214717. 
einheitliche Verbindungen aufgefaßt werden. Daß aber in Wirklichkeit Arsenverbindungen unter Umständen im Organismus mit Abbauprodukten des Eiweißmoleküls reagieren, beweist die Tatsache, daß die kürzlich von A. M i c h a e lis dargestellte p-Arsenobenzoesäure

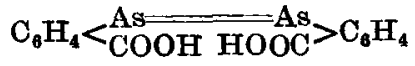

den tierischen Organismus zum Teil als 1-Arsinsäure-4-benzoylglykokoll, oder Hippurarsinsäure

wieder verläßt.

$$
\mathrm{C}_{6} \mathrm{H}_{4}<\underset{\mathrm{CO} . \mathrm{NHH}_{2}}{\mathrm{AsO}_{2} \mathrm{CH}_{2}} \cdot \mathrm{COOH}
$$

Zweck vorliegender Studie ist, mehrere solcher esterartigen Verbindungen von Aminosäuren des Eiweißmoleküls mit einer aromatischen Arsenkomponente synthetisch darzustellen und deren chemisches Verhalten zu studieren. Auch einige Arsenlipoide, als deren Lipoidkomponente höhere Alkohole gewählt wurden, sind beschrieben.

Die einfachste aromatische Arsin-Karbonsäure ist die schon vor vielen Jahren zuerst von $\mathrm{Mi}$ o ha $\mathrm{el}$ is dargestellte und von L a Coste $\mathrm{e}^{1}$ ) näher studierte p-Benzarsinsäure. Von niederen Oxydationsstufen dieser Säure sind ferner beschrieben die p-benzarsenige Säure ${ }^{1}$ ) und die p-Arsenobenzoesäure ${ }^{2}$ ). Im folgenden sind noch beschrieben das p-Benzarsenoxyd und die Phenylarsinkarbonsäure, so daß von dieser Reihe bekannt sind:
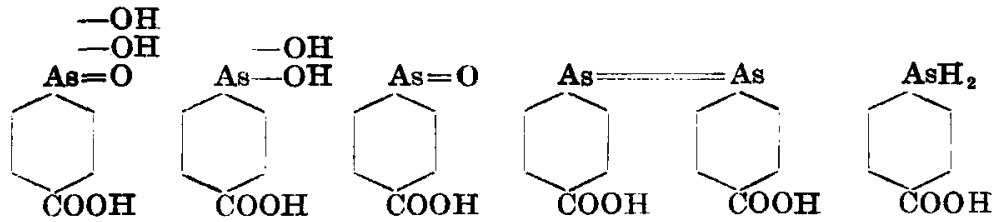

Die nächst niecieren Oxydationsstufen der Phenylarsinsäure $\mathrm{C}_{6} \mathrm{H}_{5} \mathrm{AsO}_{3} \mathrm{H}_{2}$, der Oxyphenylarsinsäure

$$
\mathrm{C}_{6} \mathrm{H}_{4}<{ }_{\mathrm{OH}}^{\mathrm{AsO}_{3} \mathrm{H}_{3}},
$$

der Amidophenylarsinsäure

der Phenylglycinarsinsäure

$$
\mathrm{C}_{6} \mathrm{H}_{4}<\mathrm{NH}_{2} \mathrm{AsO}_{3} \mathrm{H}_{2},
$$

$$
\mathrm{C}_{6} \mathrm{H}_{4}<\mathrm{NH}_{\mathrm{NHO}}^{\mathrm{AsH}_{2} \mathrm{COOH}},
$$

1) La Coste, Ann. 208, 1 (1881).

2) Michaelis, Ber. 48, 870 (1915).

Arch. d. Pharm. OCLIV. Bds. 3. Heft. 
der 3-Amido-4-oxyphenylarsinsäure u. a. sind nur in Form des Oxyds $-\mathrm{As}=\mathrm{O}$ bekannt. Es ist hier in der Tat nicht gelungen, diese Oxydstufe durch Hydratation in - $\mathrm{As}(\mathrm{OH})_{2}$ zu verwandeln. Sobald aber in das aromatische Radikal eine elektronegative Gruppe, wie $\mathrm{NO}_{2}$ oder $\mathrm{COOH}$, eingeführt wird, sind solche Verbindungen als Derivate der hypothetischen hydrierten $\mathrm{As}(\mathrm{OH})_{3}$ sehr beständig'). Deshalb entsteht auch nach L a Cost $\theta$ bei Reduktion der Benzarsinsäure mittels Jodwasserstoff und rotem Phosphor zu Benzarsenjodür

\section{$\mathrm{C}_{6} \mathrm{H}_{4}<{ }_{\mathrm{COOH}}^{\mathrm{J}_{2}}$}

Auflösen des Produktes in Natriumkarbonat und Ausfällen mit Salzsäure, nicht das Benzarsenoxyd, sondern benzarsenige Säure.

Bei der biologischen Prüfung ${ }^{2}$ ) stellte sich nun heraus, daß zwei Präparate, eins, ein mit „benzarsenige Säure" bezeichnetes amorphes weißes Pulver aus der Michaelis'schen Sammlung, und ein anderes, nach den Angaben von $\mathrm{M} \mathrm{i} \mathrm{c} \mathrm{h} \mathrm{a} \mathrm{e} \mathrm{lis} \mathrm{selbst} \mathrm{dargestelltes,}$ in langen farblosen Nadeln krystallisierendes Produkt, gewaltige Unterschiede im Tierversuche hinsichtlich Toxizität zeigten, indem das letztere sich mindestens zehnmal ungiftiger erwies. Bei wiederholter Neudarstellung zeigte sich weiter, daß der nach Auflösen des Benzarsenjodürs in Natriumkarbonat und Ausfällen mit Säure erhaltene Körper sich spielend in Aether löste und übereinstimmende quantitative Giftigkeit mit der M i c h a e l i s'schen „benzarsenigen Säure" zeigte, während er nach Kochen mit Wasser bis zur Lösung beim Erkalten in schönen langen Nadeln auskrystallisierte und nicht mohr ätherlöslich war. Die Analyse zeigte, daß die amorphe Substanz um ein Minus von $\mathrm{H}_{2} \mathrm{O}$ gegenüber der krystallisierten differierte:

$$
\begin{aligned}
& 0,1477 \mathrm{~g} \text { Substanz gaben } 0,1078 \mathrm{~g} \mathrm{Mg}_{2} \mathrm{As}_{2} \mathrm{O}^{?} \text {. } \\
& 0,1800 \mathrm{~g} \quad, \quad, \quad 0,2624 \mathrm{~g} \mathrm{CO}_{2} \text { und } 0,0400 \mathrm{~g} \mathrm{H}_{2} \mathrm{O} \text {. } \\
& 0,1372 \mathrm{~g} \quad, \quad, \quad 0,1974 \mathrm{~g} \mathrm{CO}_{2} \text { und } 0,0310 \mathrm{~g} \mathrm{H}_{2} \mathrm{O} \text {. }
\end{aligned}
$$

Berechnet für:

$\begin{array}{lccc} & \text { Gefunden: } & \mathrm{C}_{6} \mathrm{H}_{4}<\mathrm{COOH}: & \mathrm{C}_{6} \mathrm{H}_{\mathbf{6}}<\underset{\mathrm{COOH}}{\mathrm{As}(\mathrm{OH})_{8}} \text { : } \\ \text { As } & \mathbf{3 5 , 2 3} & \mathbf{3 5 , 3 8} & \mathbf{3 2 , 6 1 \%} \\ \mathrm{C} & \mathbf{3 9 , 7 4} \text { und } 39,24 & \mathbf{3 9 , 6 2} & \mathbf{3 6 , 5 2 \%} \\ \mathrm{H} & \mathbf{2 , 4 9} \text { und } \mathbf{2 , 5 3} & \mathbf{2 , 3 6} & \mathbf{3 , 0 4 \%}\end{array}$

Selbst als Salz in wässeriger Lösung ist das Benzarsinoxyd längere Zeit haltbar, ohne sich in die Hydratform umzulagern; eine unter Zusatz von Natriumkarbonat oder Natriumhydroxyd kalt

1) M ich a elis, Ann. 320, 274 (1901).

2) $S i \in b u r g, l$. e. 
bereitete wässerige mäßig konzentrierte Lösung hielt sich im Wissohrank mindestens acht Tage, ohne an Giftigkeit merkbar einzubüßen. Jedenfalls geht hieraus hervor, daß auch bei Gegenwart ausgesprochen elektronegativer Radikale im Benzolkern durch geeignete Reduktion der Arsinsäure die Arsenoxydstufe entsteht, die erst bei Gegenwart von Wasser in der Siedehitze sich zur Hydratstufe der arsenigen Säure umlagert.

Durch energische Reduktion mit Zinkstaub und Salzsäure sind verschiedene aromatische Arsinsäuren zu primären aromatischen Arsinen reduziert worden ${ }^{1}$ ). Die substituierten aromatischen Arsine, die salzbildende Gruppen im Molekül enthalten, sind im Gegensatz zum primären Phenylarsin ${ }^{2}$ ) verhältnismäßig beständige.

Zur Darstellung von Phenylarsin-4-karbonsäure wurden $5 \mathrm{~g}$ Benzarsinsäure in $100 \mathrm{ccm}$ Methylalkohol gelöst und dieser Lösung allmählich $150 \mathrm{ccm}$ Salzsäure vom spez. Gewicht 1,19 und ein Ueberschuß von Zinkstaub hinzugefügt. Man läßt die Wasserstoffentwickelung unter zeitweiligem Erwärmen auf dem Wasserbade etwa 1/2 Stunde im Gange, verdünnt den Kolbeninhalt ungefähr mit dem gleichen Volumen heißen Wassers und unterwirft bei noch bestehender Wasserstoffentwickelung der Destillation mit Wasserdampf. Das Kühlerrohr überzieht sich bald mit einer Menge schneeweißer Krystalle, die sich auch aus dem Inhalt der Vorlage abscheiden. Man tut gut in die Vorlage Aether hineinzugeben, der das Arsin löst, denn in der wässerigen Suspension färben sich die Krystalle sehr bald gelb. Die ätherische Lösung wird mit geglühtem Natriumsulfat rasch entwässert und im Exsikkator über Paraffin unter ständigem Durchleiten von Kohlensäure verdunstet. Die Substanz hinterbleibt so in Form farbloser kurzer dicker prismatischer Säulen, die in zugeschmolzener Kapillare bei $\mathbf{7 9 - 8 0 ^ { \circ }}$ schmelzen. In kaltem Wasser ist sie nur ganz wenig löslich, etwas besser in heißem Wasser, leicht in kohlensauren und ätzenden Alkalien. Ebenso löst sie sich leicht in Alkohol, Methylalkohol, Aceton, Aether, Essigäther, Eisessig und Chloroform, weniger gut in Benzol und Ligroin.

In wasserfeuchtem Zustande ist die Phenylarsin-4-karbonsäure ganz außerordentlioh luftempfindlich, indem sie sich sofort gelb färbt und dabei anscheinend in Arsenobenzoesäure übergeht. Bei längerem Aufenthalt in trockenem Zustande selbst im Vakuumexsikkator scheint sich das Arsin mit Umgehung der Arsenostufe direkt zum Arsinoxyd zu oxydieren. Die Substanz bleibt zwar

1) D. R. P. 251 571; K a h n, Chem.-Ztg. 1099 (1912).

2) Pal $\mathrm{m}$ er und $\mathrm{D}$ e h $\mathrm{n}$, Ber. 34, 3598 (1901). 
farblos, verliert aber ihr krystallines Aussehen; und setzt man ein gelindes Reduktionsmittel, z. B. phosphorige Säure zu, so wird die gelbe Arsenostufe erhalten.

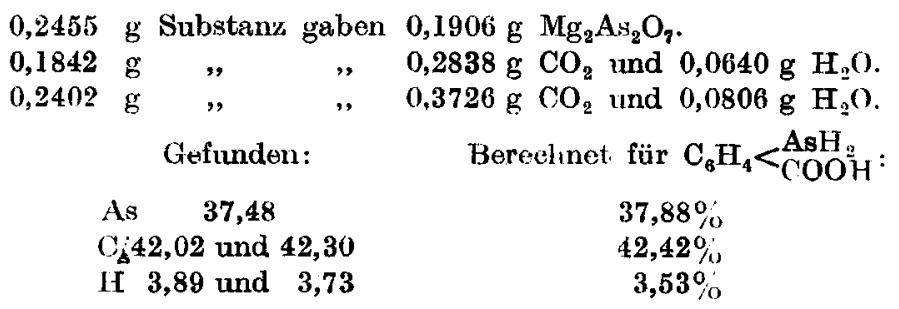

Un die zur Veresterung benötigten größeren Mengen p-Benzarsinsäure darzustellen, kann man verschiedene Wege einschlagen. Die älteste Methode besteht in der von I a Coste (1.c.) angegebenen Oxydation der Tolylarsinsäure

$$
\mathrm{C}_{6} \mathrm{H}_{4}<\mathrm{CH}_{3} \mathrm{AsO}_{3} \mathrm{H}_{2} \text {. }
$$

Diese kann mittels Kaliumpermanganat iu alkalischer Lösung durchgeführt werden, dauert aber längere Keit, und läßt auch an Ausbeute zu wünschen übrig. Bei weitem rascher und besser oxydiert man aber nach neueren Angaven von $\mathrm{M}$ i $\mathrm{c} h$ a e l i s $\mathrm{s}^{\mathbf{1}}$ ) durch zwölfstündiges Erhitzen von $3 \mathrm{~g}$ Tolylarsinsäure mit $40 \mathrm{ccm}$ Salpetersäure vom spez. Gewicht $1,2 \mathrm{im}$ Einschmelzrohr auf $150^{\circ}$. Nach eigenen Erfahrungen kann man die Dauer noch abkürzen, wenn man 3 Stunden lang auf $170^{\circ}$ erhitzt. Die Ausbeute ist hierbei fast quantitativ, die Menge der abgespaltenen Arsensäure und der gebildeten Nitroprodukte minimal.

Mich a elis und seine Sohüler benutzten früher zur-Darstellung von aromatischen Arsenverbindungen alsAusgangsmaterialien meist Arsentrichlorid und Quecksiberaryle. Die entstandenen primären Chlorarsine lassen sich dann leicht in die Arsinsäuren überführen. So wurde auch die Tolylarsinsäure dargestellt. Ganz wesentlich vereinfachen läßt sich diese Darstellung unter Anwendung der B a r t'schen Reaktion ${ }^{2}$ ). Nach dieser läßt sich eine Diazogruppe durch den Arsensäurerest ersetzen. Im vorliegenden Falle - zur Darstellung der o-Tolylarsinsäure nach diesem Verfahren bereits von P. K a r r e r angewand $\mathrm{t}^{3}$ ) - diazotiert man p-Toluidin in salzsaurer Lösung und läßt auf das gebildete Dįazotoluolchlorid Natrium-

I) M i chanlis, Ann. 320.303 (1!101).

2) D. R. P. 250264 (1912).

3) Ka r rer, Rer. 48, 310 (1915). 
arsenit einwirken. Bei Gegenwart von freiem Alkali orfolgt dann der Austausch der Diazogruppe gegen $-\mathrm{AsO}_{3} \mathrm{H}_{2}$.

Die Anwendung der $\mathrm{B}$ a r t'schen Reaktion lieferte aber, um von der p-Amidobenzoesäure direkt zur p-Benzarsinsäure zu gelangen, in diesem Falle so schlechte Ausbeute, daß sie hier, als zu wenig rationell, nicht zur Darstellung größerer Mengen verwandt wurde.

Gute Resultate erzielt man unter Verwendung der S a n dme y e r'schen Reaktion, die bei Arsenverbindungen von $A$. B e r t h e $\mathrm{i}^{1}$ ) benutzt wurde. Man geht hier von der Arsanilsäure, oder dessen käuflichem Natriumsalz, dem Atoxyl, aus, diazotiert, läßt. Kuprocyanürcyankalium einwirken und verseift das entstandene Nitril der p-Benzarsinsäure. Das etwas kostspielige Ausgangsmaterial läßt jedoch dies Verfahren, wo es sich um Darstellung uroßer Mengen handelt, nicht für jedermann geeignet erscheinen.

Bereits L a Cos te machte die Beobachtung, daß Phosphortrichlorid mit p-Benzarsinsäure unter Bildung von Krystallen reagiert. Er untersuchte diese nicht weiter, gibt aber an, daß sie durch Behandlung mit Wasser benzarsenige Säure liefern.

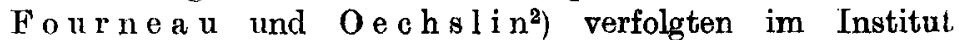
Pasteur in Paris diese Reaktion weiter und gelangten schließlich zum Dichlorarsinbenzoylchlorid

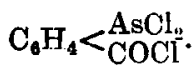

Mit Hilfe dieses Körpers führten sie dainn analog der so fruchtbaren Sohotten-Ba uman'schen Reaktion verschiedene Synthesen aus.

Die Darstellung des Dichlorarsinbenzoylchlorids gestaltet sich etwas modifiziert nach $\mathrm{F}$ o u $\mathrm{r}$ e a $\mathrm{u}$ und $\mathrm{O}$ e ch $\mathrm{s}$ i in folgendermaßen.

$\mathrm{Zu}$ einer Suspension von $250 \mathrm{~g}$ Benzarsinsäure in $500 \mathrm{~g}$ Chloroforn gibt man unter guter Eiskühlung in kleinen Portionen eine Lösung von $280 \mathrm{~g}$ Phosphortrichlorid in $500 \mathrm{~g}$ Chloroform. Die Reaktion beginnt spontan unter Wärmeentwickelung. Schüttelt man häufig um, so geht die Benzarsinsäure allmählich und fast völlig in Lösung und an ihrer Stelle durchsetzt sich der Kolbeninhalt mit großen, fast farblosen Krystallen. Man beendet die Reaktion, indem man einige Minuten auf dem Wasserbad unter Rückflußkühlung im Sieden erhält. Es resultiert eine klare Lösung, während am

1) B ertheim, Ber. 41, 1857 (1908).

2) Fournea und $O e c h s l i n$, Bull. de la Soc. chim. de France, XI, 909 (1912). 
Boden des Gefäßes sich Schmieren von phosphoriger Säure abgesetzt haben. Man läßt völlig erkalten bis zur Abscheidung eines reichlichen Krystallbreis und fügt auf einmal $210 \mathrm{~g}$ Phosphorpentachlorid hinzu, wodurch die weitere Reaktion beginnt, und die Krystalle unter Wärmeentwickelung sich lösen. Man hält noch etwa eine Stunde am Rückflußkühler in schwachem Sieden, legt dann den Kühler um und destilliert das Lösungsmittel und den Ueberschuß der Reagentien mittels Wasserbad ab. Das gesuchte Chlorid wird dann bei 10-12 mm Druck destilliert, wobei es zwischen 187 und $189^{\circ}$ übergeht. Wendet man ein geringeres oder gar kein Vakuum an, so verkohlt der Inhalt des -Fraktionierkolbens leicht bei sehr viel niedriger Temperatur.

Das Dichlorarsinbenzoylchlorid bildet eine fast farblose, nur wenig opaleszierende, besonders an feuchter Luft rauchende Flüssigkeit. Mit Chloroform, Benzol, Aether usw. ist es in jedem Verhältnis mischbar. Mit Wasser entsteht ein krümeliger Niederschlag. Von ätzenden Alkalien wird es bei längerem Schütteln gelöst. Geschieht diese Lösung ohne Anwendung von Wärme, so wird durch Säure das amorphe Benzarsinoxyd gefällt, anderenfalls die gut ausgeprägten Krystalle der benzarsenigen Säure.

Fourneau und $\mathrm{Oechslin}$ ließen dieses Chlorid auf Alkohole (Aethylalkohol und Guajakol) einwirken und erhielten so zunächst das Oxyd des benzarsinsauren Alkyls, das durch Oxydation mit Wasserstoffsuperoxyd in alkalischer Lösung in die Arsinsäure übergeführt wurde. Von stiokstoffhaltigen Produkten stellten sie den Chinin- und Stovainäther des Benzarsinoxyds dar, oxydierten sie zu den Arsinsäuren und reduzierten letztere mittels des von Ehrlich und Bertheim angegebenen Hydrosulfitgemisches auch zu den Arsenoverbindungen.

L. H u g o une $\mathrm{q}$ und A. More $\mathbf{l}^{1}$ ) ließen das Dichlorarsinbenzoylchlorid auf eine sodaalkalische Glykokolllösung unter langdauerndem Turbinieren einwirken, wobei das Chloratom der COCl-Gruppe mit der $\mathrm{NH}_{2}$-Gruppe in Reaktion trat, während das Chlorarsin sich zu Arsinoxyd umbildete. Das durch Säurezusatz ausgeschiedene Arsinoxydbenzoylglykokoll wurde durch Wasserstoffsuperoxyd zur Hippurarsinsäure oxydiert und diese mit Hydrosulfit zu Arsenohippursäure reduziert.

Die krystallinische Hippurarsinsäure HOOC. $\mathrm{CH}_{2} \mathrm{NH} . \mathrm{CO}$. $\mathrm{C}_{6} \mathrm{H}_{4} \cdot \mathrm{AsO}_{3} \mathrm{H}_{2}$ ist nach diesen Autoren spielend löslich in Wasser,

1) H u g o u nenqund M orel, Journ. de Pharm. et de Chim. VII, 383 (1913). 
gut auch in Methyl- und Aethylalkohol, fast unlöslich in Fettlösungsmitteln. Wie andere Arylarsinsäuren gibt sie erst beim Erwärmen der wässerigen Lösung einen Niederschlag mit Calciumchlorid oder Magnesiamixtur. Sie schmilzt nicht ohne Zersetzung.

Die Arsenohippursäure

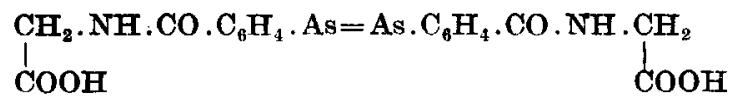

ist ein gelbes Pulver, das in jeder Beziehung den Arsenoverbindungen gleicht, entsprechend seiner saueren Funktion aber die Figenschaft hat, sich in ätzenden und kohlensauren Alkalien zu lösen. Die Auflösungen in diesen Medien halten sich aber schlecht und setzen, selbst wenn man sie vor Luft geschützt hält, einen weißlichen Niederschlag ab, der sehr giftige Arsenoxyde enthält.

Die Anwendung von Natriumkarbonat bei der Veresterung der Benzoylchloridgruppe mit der Aminogruppe anderer Aminosäuren lieferte aber nur sehr bescheidene Ausbeuten. Ausgezeichnet, wurden die Resultate, als das nach dem Vorgange von Emil $\mathrm{Fis} \mathrm{cher1}$ zur Benzoylierung von Aminosäuren verwandte Natriumbikarbonat bei einem erheblichen Ueberschuß von Säurechlorid herangezogen wurde.

Die auf diese Weise zunächst erhaltenen Arsenoxyde vom Typ

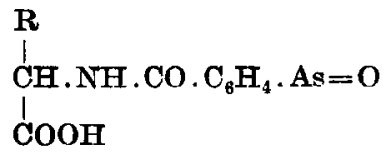

sind in ihren Eigenschaften sich sehr ähnlich. Keine dieser Substanzen - auch nicht ihre Oxydations- und Reduktionsprodukte besitzt einen scharfen Schmelzpunkt; nicht einmal die Temperatur, bei der sich die Körper zu zersetzen beginnen, kann zur Identifizierung herangezogen werden; kleine Variationen in der Menge der in die Kapillare eingebrachten Substanz, sowie die Art des Erhitzens lassen selbst den Beginn des Erweichens unter Dunkelfärbung stark differieren. Alle diese Arsinoxyde sind amorphe weiße Pulver, die in Alkohol und Methylalkohol leicht löslich sind, sehr viel weniger in Aether, nur spurweise in Chloroform und Kohlenwasserstoffen. Infolge des Einflusses der Karboxylgruppe lösen sie sich außer in ätzenden Alkalien auch in Karbonaten und Bikarbonaten. Unlöslich sind sie in verdünnter Salzsäure. Es gelang auch nicht nach den

1) E. F i s c h $\theta$ r, Ber. 32, 2451 (1899). 
üblichen Methoden den Sauerstoff der As=0-Gruppe durch Halogen oder Schwefel zu ersetzen.

Durch vorsichtige Oxydation mittels Wasserstoffsuperoxyd in alkalischer Lösung lassen sich diese Arsinoxyde leicht und glatt zu gut krystallisierenden Arsinsäuren oxydieren, ohne daß dabei die Aminosäurekomponente merkbar angegriffen wird.

Bei der Reduktion der Arsinoxyde zu den Arsenoverbindungen bewährte sioh Natriumamalgam am besten. Die gelbe Farbe dieser Reduktionsprodukte nüanzierte nicht nur bei den verschiedenen Arsenokörpern etwas, sondern oft auch bei der wiederholten Neudarstellung ein und derselben Substanz. Die Menge des Lösungsmittels, in dem die Reduktion vorgenommen wird, die Temperatur, sowie die Art des Trocknens scheinen hier von Einfluß zu sein.

Die zur Anwendung gekommenen Aminosäuren waren sämtlich die synthetisch dargestellten, optisch inaktiven, Körper.

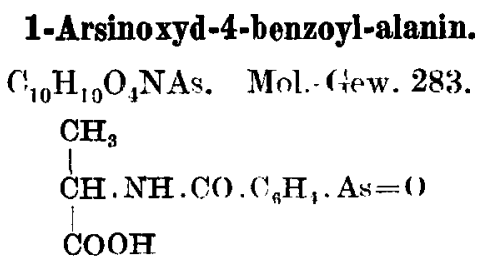

2,2 $\mathrm{g}$ Alanin (1/40 Mol.) werden in $100 \mathrm{ccm}$ Wasser suspendiert. und mit $35 \mathrm{~g}$ Natriumbikarbonat versetzt. Dann werden in Zeitabständen von etwa 30 Minuten in 10 Portionen $21,4 \mathrm{~g}$ Dichlorarsinbenzoylchlorid $\mathbf{8} / 40 \mathrm{Mol}$.) hinzugegeben und jedesmal auf der Maschine geschüttelt, bis das Chlorid unter Kohlensäureentwickelung in Lösung gegangen ist. Es ist dabei nicht nötig, eine besonders niedere Temperatur einzuhalten, gegen Ende der Reaktion tut man sogar gut, die Temperatur auf $35-40^{\circ}$ zu steigern. Ist alles Chlorid bis auf geringe Trübungen in Lösung gegangen und ebenso alles Bikarbonat verbraucht, so wird filtriert, auf $0^{0}$ abgekühlt und mit $110 \mathrm{com}$ gleichfalls eiskalter 5-Normal-Salzsäure versetzt. Der Niederschlag wird scharf abgesaugt, wiederholt mit kaltem Wasser ausgewaschen und dann öfter mit nicht zu geringen Mengen Aether digeriert, um das nicht veresterte Benzarsinoxyd herauszulösen. Ausbeute an trockenem Arsinoxydbenzoylalanin 5,2 $\mathrm{g}=74 \%$ der Theorie.

Die amorphe weiße Substanz ist ebenso wie die im folgenden beschriebenen Arsenoxyde unlöslich in Wasser, leicht löslich in Alkalien und Alkohol, nicht ganz unlöslich in Aether. 
E. Sieburg: Ester aromatischer Arsenverbindungen.

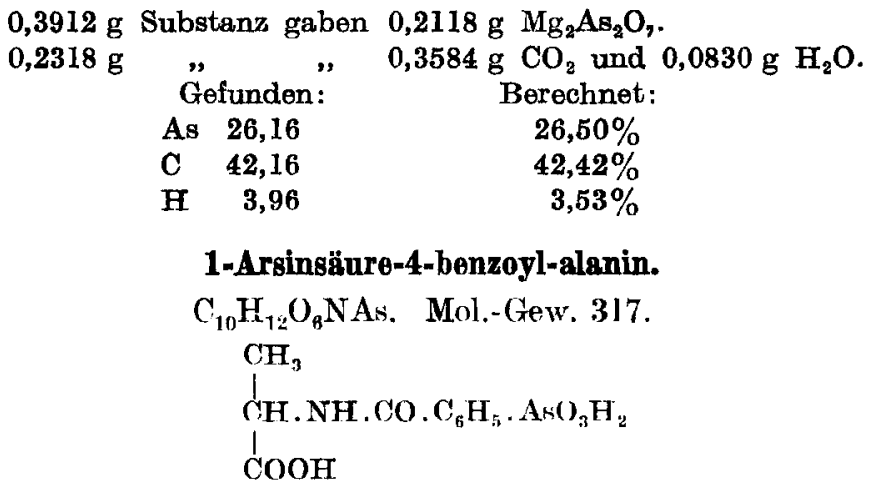

Das Arsinoxyd des Benzoylalanins wird in einem Ueberschuß von Normal-Natronlauge gelöst und mit einigen Tropfen 30\%igem Wasserstoffsuperoxyd versetzt. Nach etwa dreistündigem Stehen in Eiswasser wird mit einer entsprechenden Menge Normal-Salzsäure ausgefällt und nach Stehenlassen über Nacht das ausgefallene Krystallmehl abgesaugt, mit kaltem Wasser ausgewaschen und aus heißem Wasser umkrystallisiert.

Die Säure bildet winzige kubische Kryställohen, die sioh sohwer in kaltem, leichter in heißem Wasser lösen. Mit Calciumchlorid oder mit Magnesiamixtur bleibt die Lösung ihrer Alkalisalze zunächst klar, trübt sich aber beim Kochen. Mit Kupfersalzen bilden sich grüne Niederschläge, die mit wenig Ammoniak mit tiefblauer Farbe in Lösung gehen. Nach einiger Zeit krystallisieren aus dieser Lösung wundervoll lasurblaue lange Nadeln, die arsen- und ammoniakhaltig sind. Mit anderen Schwermetallsalzen gibt die Arsinsäure Niederschläge, die ganz unlöslich sind. Analog verhalten sich die gleich zu beschreibenden Arsinsäuren des Benzoylphenylalanins, des Benzoyltyrosins und des Benzoylleucins.

$0,1828 \mathrm{~g}$ Substanz gaben bei $17^{\circ}$ und $770 \mathrm{~mm} 7,0 \mathrm{cem} N$.

$\begin{array}{cc}\text { Gefunden: } & \text { Berechnet: } \\ \text { N } 4,50 & 4,41 \%\end{array}$

1-Arseno-4-benzoylalanin. $\left(\mathrm{C}_{10} \mathrm{H}_{10} \mathrm{O}_{3} \mathrm{NAs}\right)_{2}$. Mol.-Gew. 534 .<smiles>CC(NC(=O)C(C)NC=CCCCCCCCCCCCC(=O)O)C(=O)O</smiles>

Arsinaxydbenzoylalanin wird in Wasser suspendiert und mit dem 1 1/2fachen der berechneten Menge 2,5\%igem Natriumamalgam 
versetzt. Unter Kühlung mit Wasser wird solange sanft geschüttelt, bis das Oxyd völlig in Lösung gegangen und die Wasserstoffentwickelung beendet ist. Die vom Quecksilber abgegossene klare gelbe Lösung wird mit Essigsäure übersättigt, der Niederschlag abgesaugt und ausgewaschen und im Vakuum über Schwefelsäure getrocknet.

Die amorphe gelbe Substanz ist unlöslich in Wasser, sehr schwer: löslich in Alkohol, leicht löslich in ätzenden und kohlensauren Alkalien.

$0,2834 \mathrm{~g}$ Suhstanz gaben bei $14^{\circ}$ und $770 \mathrm{~mm} 13,0 \mathrm{ccm} \mathrm{N}$.

$\begin{array}{cc}\text { Gefunden: } & \text { Berechnet: } \\ \mathbf{5 , 4 7} & \mathbf{5 , 2 4} \%\end{array}$

1-Arsinoxyd-4-benzoyl-phenylalanin.

$$
\begin{aligned}
& \mathrm{C}_{16} \mathrm{H}_{14} \mathrm{O}_{4} \mathrm{NAs} . \quad \mathrm{Mol} \text { - Gew. } 359 . \\
& \mathrm{CH}_{2} \cdot \mathrm{C}_{6} \mathrm{H}_{5} \\
& \mathrm{CH} . \mathrm{NH} . \mathrm{CO} . \mathrm{C}_{6} \mathrm{H}_{4} \cdot \mathrm{As}=\mathrm{O} \\
& \mathrm{COOH}
\end{aligned}
$$

Die Darstellung erfolgte wie heim Alaninderivat angegeben; es wurden verwendet:

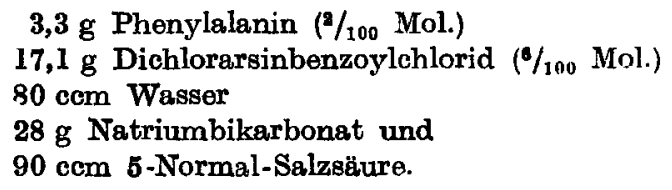

Die Ausbeute betrug $5,8 \mathrm{~g}=\mathbf{8 2} \%$ der Theorie. Die Substanz bildet getrocknet ein feines weißes in Alkohol und wässerigem Alkali lösliches Pulver.

$\begin{array}{ccc}0,3779 \mathrm{~g} & \text { Substanz gaben } & 0,1606 \mathrm{~g} \mathrm{Mg}_{2} \mathrm{As}_{2} \mathrm{O}_{\eta} . \\ 0,1972 \mathrm{~g} & \# & 0,3900 \mathrm{~g} \mathrm{CO} \text { und } 0,0714 \mathrm{~g} \mathrm{H}_{0} \mathrm{O} . \\ \text { Gefunden: } & \text { Berechnet: } \\ \text { As } 20,52 & 20,89 \% \\ \text { C } & \mathbf{5 3 , 9 4} & 53,48 \% \\ \text { H } & 3,95 & 3,90 \%\end{array}$

1-Arsinsäure-4-benzoyl-phenylalanin.

$$
\begin{aligned}
& \mathrm{C}_{16} \mathrm{H}_{18} \mathrm{O}_{6} \mathrm{NAs} . \mathrm{Mol} \text { - Gew. } 393 . \\
& \mathrm{CH}_{2} \cdot \mathrm{C}_{6} \mathrm{H}_{5} \\
& \vdots \mathrm{CH} . \mathrm{NH} . \mathrm{CO} \cdot \mathrm{C}_{6} \mathrm{H}_{4} \cdot \mathrm{AsO}_{3} \mathrm{H}: \\
& \mathrm{COOH}
\end{aligned}
$$

wurde erhalten durch Oxydation des Arsinoxyds mittels Wasserstoffsuperoxyd in alkalischer Lösung, und bildet in Alkohol und heißem Wasser lösliche Nädelchen. 
$0,1965 \mathrm{~g}$ Substanz gaben bei $17^{\circ}$ und $773 \mathrm{~mm} 5,6 \mathrm{ccm} \mathrm{N}$.

$\begin{array}{cc}\text { Gefunden: } & \text { Berechnet: } \\ \mathbf{N} 3, \mathbf{3 6} & \mathbf{3 , 5 6 \%} .\end{array}$

1-Arseno-4-benzoyl-phenylalanin. $\left(\mathrm{C}_{16} \mathrm{H}_{14} \mathrm{O}_{3} \mathrm{NAs}\right)_{2}$. Mol.-Gew. 686 .<smiles>O=C(CCCCCCC(=O)NC(Cc1ccccc1)C(=O)O)NC(Cc1ccccc1)C(=O)O</smiles>

wurde erhalten durch Reduktion des Arsenoxyds mit Natriumamalgam. Das gelbe Pulver löst sich in wässerigem Alkali.

$0,2216 \mathrm{~g}$ Substanz gaben bei $12^{\circ}$ und $768 \mathrm{~mm} 7,7 \mathrm{ccm} \mathrm{N}$.
Gefunden:
Berechnot:
N $4,17 \quad 4,08 \%$
1-Arsinoxyd-4-benzoyl-tyrosin.
$\mathrm{C}_{16} \mathrm{H}_{14} \mathrm{O}_{5} \mathrm{NAs}$. Mol.-Gew. 375.
$\mathrm{CH}_{2} \cdot \mathrm{C}_{6} \mathrm{H}_{4} \cdot \mathrm{OH}$
L
CH. NH. CO. $\mathrm{C}_{6} \mathrm{H}_{4} \cdot \mathrm{A}:=$ )
COOH

Die Veresterung bei Gegenwart von Natriumbikarbonat fuhrte hier nicht zum Ziel. Beim Benzoylieren von Amido-oxy-säuren in schwach alkalischer Lösung wird sowohl in der $\mathrm{NH}_{2}$ - als auch in der OH-Gruppe der Benzoylrest eingeführt, und man erhält vorwiegend Dibenzoylverbindungen, oder wenigstens ein Gemisch von Dibenzoyloxyamidosäuren mit Monobenzoylsubstituten. Man kann nun erstere durch längeres Behandeln mit Alkali in der Wärme in die Monobenzoylamidooxysäuren zurückverwandeln. Arbeitet man dagegen nach dem Vorbilde von Sørensen und A n d e r $\left.\operatorname{sen}^{1}\right)$ von vornherein in ausgesprochen alkalischer Lösung und sorgt dafür, daß die Benzoylierungsflüssigkeit auch am Schlusse des Prozesses noch ungefähr $1 / 2$-normal alkalisch ist, so erhält man nur monobenzoylierte Produkte.

$8,25 \mathrm{~g}$ Tyrosin (1/20 Mol.) werden in $12,5 \mathrm{ccm}$ Wasser und 37,5 com 2-Normal-Natronlauge gelöst. Die Flüssigkeit ist dann $1 / 2$-normal alkalisch. In einem Meßzylinder werden dann $57 \mathrm{~g}$

1) S. P. L. Seren sen und A. C. Andersen, Compt. rend. des trav. du Lab. de Carlsberg, VII, 85 (1908). 
Dichlorarsinbenzoylchlorid und in einem zweiten $720 \mathrm{ccm}$ 2-NormalNatronlauge gebracht und von beiden entsprechende Mengen in 12 Portionen in die Tyrosinlösung gegeben. Das Gemisch wird dann jedesmal etwa 20 Minuten auf der Maschine geschüttelt und vor jedem neuen Zusatz durch Einsetzen in eine Kältemischung gut gekühlt. Nach Beendigung der Reaktion wird filtriert und das Filtrat mit $320 \mathrm{~cm} 5$ Normal-Salzsäure versetzt. Der Niederschlagr wird abgesaugt, mit Wasser gründlich gewaschen und wiederholt mit Aether digeriert. Der in Aether unlösliche Rückstand wird nochmals in der eben ausreichenden Menge 2-Normal-Natronlauge gelöst, durch Salzsäure wieder ausgefällt, gewaschen und getrocknet. Ausbeute $5 \mathrm{~g}=\mathbf{2 7} \%$ der Theorie.

Dieses Arsinoxyd-benzoyl-tyrosin, in getrocknetem Zustund ein weißliches Pulver, ist außer in Alkohol und Alkalien auch in reinem Wasser nicht ganz unlöslich.

$\begin{array}{ccc}0,1522 \mathrm{~g} & \text { Substanz gaben } & 0,0632 \mathrm{~g} \mathrm{Mg}_{2} \mathrm{As}_{2} \mathrm{O}_{7} . \\ 0,1456 \mathrm{~g} & \text { " } & 0,2750 \mathrm{~g} \mathrm{CO}_{2} \text { und } 0,0458 \mathrm{~g} \mathrm{H}_{2} \mathrm{O} . \\ \text { Gefunden: } & \text { Berechnet: } \\ \text { As } 20,11 & 20,00 \% \\ \mathrm{C} \quad 51,52 & 51,20 \% \\ \text { H } \quad 3,52 & 3,74 \%\end{array}$

\section{1-Arsinsäure-4-benzoyl-tyrosin.}

$\mathrm{C}_{16} \mathrm{H}_{16} \mathrm{O}_{7}$ NAs. Mol.-Gew. 409.

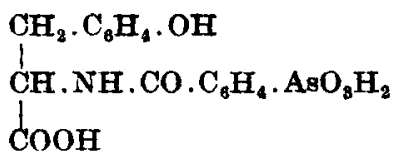

dargestellt durch Oxydation des vorigen Arsinoxyds mittels Wasserstoffsuperoxyd. Die Säure bildet längliche zugespitzte Plättchen und ist außer in Alkalien, Alkohol und heißem Wasser auch in kaltem Wasser merkbar löslich.

$0,2118 \mathrm{~g}$ Substanz gaben bei $15^{\circ}$ und $749 \mathrm{~mm} 6,6 \mathrm{~cm} \mathrm{~N}$.

$$
\begin{array}{cc}
\text { Gefunden: } & \text { Berechnet: } \\
\text { N } 3,59 & 3,42 \% .
\end{array}
$$

1-Arseno-4-benzoyl-tyrosin. $\left(\mathrm{C}_{16} \mathrm{H}_{14} \mathrm{O}_{4} \mathrm{NAs}\right)_{2}$. Mol.-Gew. 718 .

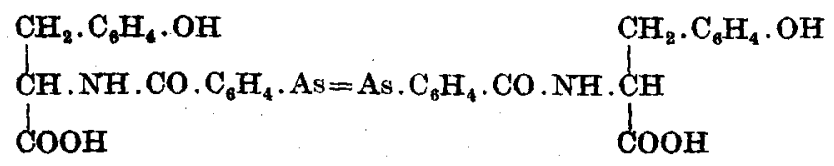


dargestellt aus dem Oxyd wie gewöhnlich, bildet ein in wässerigem Alkali lösliches gelbes Pulver.

$0,2006 \mathrm{~g}$ Substanz gaben bei $17^{\circ}$ und $752 \mathrm{~mm} 7,0 \mathrm{~cm} \mathrm{~N}$.

$$
\begin{array}{cc}
\text { Gefunden: } & \text { Berechne } \\
\text { N } 4,01 & 3,90 \%
\end{array}
$$

Vorstehende drei Substanzen geben als Tyrosinabkömmlinge natürlich rie bekannten Tyrosinreaktionen. Die P ir ia'sche Reaktion trat am schönsten ein bei der Arsinsäure: Es wurde eine Probe mit konz. Schwefelsäure auf dem Wasserbade erwärmt, mit Wasser verdünnt, jetzt mit Baryumkarbonat neutralisiert und mit Eisenchlorid versetzt, wodurch eine längere Zeit haltbare schöne Violettfärbung hervorgerufen wurde.

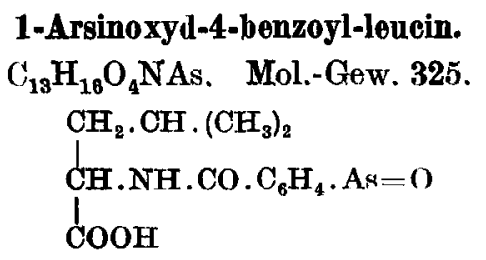

Es wurde bereitet analog dem Arsinoxyd des Benzoylalanins aus 3,9 g Leucin ( $1 / 100$ Mol.)

25,7 g Dichlorarsinbenzoylchlorid ( $\% / 100$ Mol.)

$180 \mathrm{cem}$ Wasser

$42 \mathrm{~g}$ Natriumbikarbonat und

$135 \mathrm{ccm}$ 6-Normal-Salzsäure,

und in einer Ausbeute von $7,4 \mathrm{~g}=76,4 \%$ der Theorie erhalten in Form eines weißen Pulvers, das sich in Alkohol und wässerigem Alkali löst.

$0,1635 \mathrm{~g}$ Sulostanz gaben $0,0782 \mathrm{~g} \mathrm{Mg}_{2} \mathrm{As}_{2} \mathrm{O}_{7}$.

$0,1754 \mathrm{~g} \quad, \quad, \quad 0,3064 \mathrm{~g} \mathrm{CO}_{2}$ und $0,0800 \mathrm{~g} \mathrm{H}_{2} \mathrm{O}$.

\begin{tabular}{ccc}
\multicolumn{2}{r}{ Gefunden: } & Berechnet: \\
As & $\mathbf{2 3 , 0 9}$ & $23,08 \%$ \\
C & $\mathbf{4 7 , 6 4}$ & $\mathbf{4 8 , 0 0 \%}$ \\
H & $\mathbf{5 , 4 8}$ & $\mathbf{4 , 9 2} \%$
\end{tabular}

\section{1-Arsinsäure-4-benzoyl-leuein.}

$\mathrm{C}_{13} \mathrm{H}_{18} \mathrm{O}_{6}$ NAs. Mol.-( $\mathrm{H} \in \mathrm{W}$. 359 .

$\mathrm{CH}_{2} \cdot \mathrm{CH} \cdot\left(\mathrm{CH}_{3}\right)_{2}$
$\mathrm{dH} . \mathrm{NH} \cdot \mathrm{CO} \cdot \mathrm{C}_{6} \mathrm{H}_{4} \cdot \mathrm{A} * \mathrm{O}_{3} \mathrm{H}_{2}$
(OOOH 
durch Oxydation der vorigen Substanz mit Wasserstoffsuperoxyd erhalten, bildet winzige Nädelchen, die sich leicht in Alkalien lösen, schwer dagegen in heißem Wasser.

$0,2112 \mathrm{~g}$ Substanz gaben bei $14^{0}$ und $749 \mathrm{~mm} 7,6 \mathrm{com} \mathrm{N}$.

$\begin{array}{cc}\text { Gefunden: } & \text { Berechnet: } \\ \mathrm{N} \quad 4,17 & 3,90 \%\end{array}$

1-Arseno-4-benzoyl-leucin. $\left(\mathrm{C}_{13} \mathrm{H}_{16}()_{3} \mathrm{NAs}\right)_{2}$. Mol.-(rew. blo.

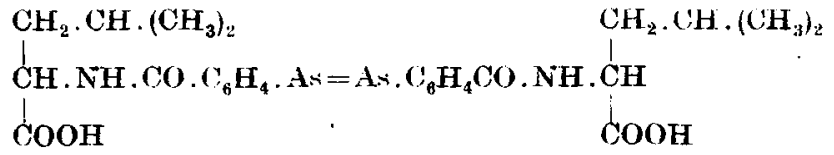

wurde durch Reduktion des Oxyds erhalten in Form eines gelben Pulvers.

$0,2755 \mathrm{~g}$ Substanz gaben bei $15^{\circ}$ und $749 \mathrm{~mm} 10,5 \mathrm{ccm} N$.

$\begin{array}{cc}\text { Gefunden: } & \text { Berechnet: } \\ \mathrm{N} \quad \mathbf{4}, 39 & \mathbf{4 , 5 3} \%\end{array}$

An dem Beispiel dieser Leucin-Arsenverbindungen wurde versucht, einen Einblick zu gewinnen in die Festigkeit, mit der die Aminosäure mit dem aromatischen Komplex verbunden ist. Die Substanzen wurden nach Absättigung ihrer saueren Gruppen noch mit ungefähr dem Vierfachen der berechneten Menge 2-Normal-Natronlauge eine bestimmte Zeit im siedenden Wasserbade gehalten und nach dem Abkühlen der Ueberschuß des freien Alkalis unter Verwendung von Phenolphthalein als Indikator zurücktitriert. Dabei wird angenommen, daß das gebundene Alkali lediglich zur Verseifung der Amidogruppen verbraucht wird, und diese proportional dem Alkaliverbrauch vor sich geht.

\section{Arsinoxy d.} $34,6 \%$.

$0,5634 \mathrm{~g}$ binden in 15 Minuten $0,6 \mathrm{ccm} \mathrm{N}$. NaOH; Verseifung zu

$0,3875 \mathrm{~g}$ binden in 60 Minuten $1,1 \mathrm{ccm} \mathrm{N} \cdot \mathrm{NaOH}$; Verseifung zu $92,2 \%$

II. Arsins äu re.

$0,6942 \mathrm{~g}$ binden in 15 Minuten $0,25 \mathrm{ccm} \mathrm{N} . \mathrm{NaOH}$; Verseifung zu $12,9 \%$.

$0,8004 \mathrm{~g}$ binden in 60 Minuten $1,20 \mathrm{ccm} \mathrm{N}$.-NaOH; Verseifung zu $53,8 \%$. 
III. A r s e noverbind ung. zu $56,3 \%$.

$0,4116 \mathrm{~g}$ binden in 15 Minuten $0,75 \mathrm{ccm} \mathbf{N}$.-NaOH; Verseifung zu $138,1 \%$.

$0,5370 \mathrm{~g}$ binden in 60 Minuten 2,40 ccm N.-NaOH; Verseifung

Es hat hiernach den Anschein, als ob die Gegenwart des stabilen Arsensäurerestes auch auf die Amidbindung einen gewissen festigenden Einfluß ausübt. $\mathrm{Da} ß$ in der Arsenostufe bei längerem Kontakt mit Alkali in der Wärme mehr als das theoretische Maximum von Alkali gebunden wird, spricht dafür, daß neben der Verseifung auch noch andere Prozesse vor sich gehen, unter denen sicherlich oxydative Veränderungen der so labilen Arsenogruppe eine Rolle spielen dürften.

\section{1-Arsinoxyd-4-benzoyl-asparaginsăure.}

$$
\begin{aligned}
& \mathrm{C}_{11} \mathrm{H}_{10} \mathrm{O}_{6} \mathrm{NAs} . \mathrm{Mol}-\mathrm{Gew} .327 . \\
& \mathrm{CH}_{2} \cdot \mathrm{COOH} \\
& \mathrm{CH} . \mathrm{NH} . \mathrm{CO} \cdot \mathrm{C}_{6} \mathrm{H}_{4} \cdot \mathrm{As}=\mathrm{O} \\
& \mathrm{COOH}
\end{aligned}
$$

wurde dargestellt aus:

\section{$3,3 \mathrm{~g}$ Asparaginsäure (1/40 Mol.)}

$21,4 \mathrm{~g}$ Dichlorarsinbenzoylehlorid ( $3 / 40$ Mol.)

$100 \mathrm{ccm}$ Wasser

35 g Natriumbikarbonat und

$110 \mathrm{ccm}$ 5-Normal-Salzsäure,

und in einer Ausbeute von $5,6 \mathrm{~g}=68 \%$ der Theorie erhalten. Dieses Arsinoxyd ist auch schon in kaltem Wasser etwas löslich.

$$
\begin{array}{ccc}
0,1936 \mathrm{~g} & \text { Substanz gaben } & 0,0928 \mathrm{~g} \mathrm{Mg}_{2} \mathrm{As}_{2} \mathrm{O}_{7} . \\
0,1288 \mathrm{~g} & \text { " } & 0,1890 \mathrm{~g} \mathrm{CO}_{2} \text { und } 0,0371 \mathrm{~g} \mathrm{H}_{2} \mathrm{O} . \\
\text { Gefunden: } & \text { Berechnet: } \\
\text { As } 23,14 & 22,93 \% \\
\mathrm{C} \quad 40,02 & 40,36 \% \\
\mathrm{H} \quad 3,23 & 3,06 \%
\end{array}
$$

1-Arsinsäure-4-benzoyl-asparaginsäure.

$$
\begin{aligned}
& \mathrm{C}_{11} \mathrm{H}_{12} \mathrm{O}_{8} \mathrm{NAs} \text {. Mol.-Gew. } 361 . \\
& \mathrm{CH}_{2} \cdot \mathrm{COOH} \\
& \mathrm{CH} . \mathrm{NH} . \mathrm{CO} \cdot \mathrm{C}_{6} \mathrm{H}_{4} \cdot \mathrm{AsO}_{3} \mathrm{H}_{2} \\
& \mathrm{COOH}
\end{aligned}
$$

Diese Säure ist recht beträchtlich auch in kaltem Wasser löslich. Man muß deshalb bei der Darstellung nach der Oxydation 
des Arsinoxyds die Lösung erheblich einengen. Nach längerem Stehen im Eisschrank fällt dann die Säure in wetzsteinförmigen Krystallen, die oft zu drusigen Aggregaten angeordnet sind, aus. Sie bläut Kongofarbstoff.

$0,2372 \mathrm{~g}$ Substanz gaben bei $17^{\circ}$ und $746 \mathrm{~mm} 8,4 \mathrm{~cm} \mathrm{~N}$.
Gefunden:
N 4,08
Berechnet:
$\mathbf{3 , 8 8} \%$

1-Arseno-4-benzoyl-asparaginsäure.

$\left(\mathrm{C}_{11} \mathrm{H}_{10} \mathrm{O}_{5} \mathrm{NAs}\right)_{2}$. Mol.-Gew. 622 .

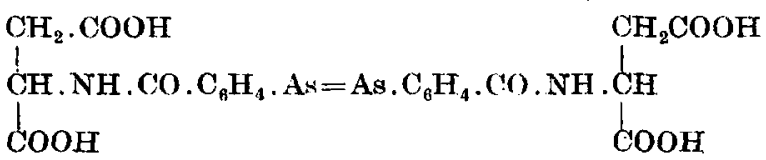

$0,1746 \mathrm{~g}$ Substanz gaben bei $18^{\circ}$ und $746 \mathrm{~mm} 6,8 \mathrm{~cm} \mathrm{~N}$.

$\begin{array}{cc}\text { Gefunden: } & \text { Berechnet: } \\ \mathrm{N} 4,41 & 4,50 \%\end{array}$

Der Körper, ein gelbes Pulver wie die übrigen Arsenoverbindungen, ist auch ohne Alkalizusatz, wenn auch nur wenig, in Wasser löslich.

dargestellt aus:

1-Arsinoxyd-4-benzoyl-glutaminsäure. $\left(\mathrm{C}_{12} \mathrm{H}_{12} \mathrm{O}_{6}\right.$ NAs. Mol.-Gew. 341.

$\left(\mathrm{CH}_{2}\right)_{2} \cdot \mathrm{COOH}$

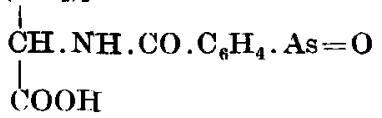

$3,6 \mathrm{~g}$ Glutaminsäure ( $1 / 40$ Mol.)

21,4 g Dichlorarsinbenzoylehlorid ( $1 / 40$ Mol.)

$100 \mathrm{ccm}$ Wasser

$35 \mathrm{~g}$ Natriumbikarbonat und

$110 \mathrm{ccm}$ 5-Normal-Salzsäure,

und in einer Ausbeute von $4,7 \mathrm{~g}=55 \%$ der Theorie erhalten. Bei längerem Stehen an der Luft hat dieser Körper die Tendenz, sich in eine glasige Masse zu verwandeln.

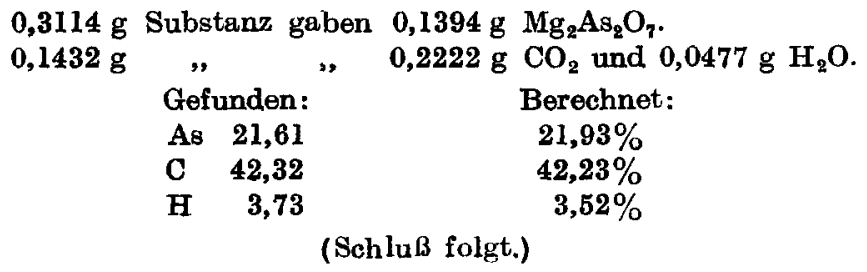


E. Sieburg: Ester aromatischer Arsenverbindungen.

1-Arsinsäure-4-benzoyl-glutaminsäure. $\mathrm{C}_{12} \mathrm{H}_{14} \mathrm{O}_{8}$ NAs. Mol.-Gew. 375 .

$\left(\mathrm{CH}_{2}\right) \cdot \mathrm{COOH}$

1 $\mathrm{CH} . \mathrm{NH} \cdot \mathrm{CO} \cdot \mathrm{C}_{6} \mathrm{H}_{4} \cdot \mathrm{AsO}_{3} \mathrm{H}_{2}$ coOH

Diese Säure, ebenfalls Kongo bläuend, ist in jedem Verhältnis in Wasser löslich. Zur Darstellung wurde wie gewöhnlich das Oxyd mit Wasserstoffsuperoxyd in alkaliseher Lösung oxydiert, die Natronlauge mit einer entsprechenden Menge Salzsäure abgesättigt, und die Mutterlauge im Vakuum bei gelinder Wärme zur Trockne verdunstet. Die Arsinsäure wurde dem Rückstand mit starkem Alkohol entzogen und aus diesem mit Ligroin ausgefällt. Beim Stehen an der Luft bildet sich alsbald ein farbloser Sirup, aus dem beim längeren Aufbewahren im Exsikkator einige kubische Kryställchen sich abscheiden, ohne daß es gelungen ist, den ganzen Sirup spontan zur Krystallisation zu bringen. Zur Analyse wurde die Substanz einige Stunden im Vakuum über Schwefelsäure bei $70^{\circ}$ gehalten.

$0,2276 \mathrm{~g}$ Substanz gaben bei $16^{\circ}$ und $750 \mathrm{~mm} 7,7 \mathrm{ccm} \mathrm{N}$. Gefunden:

$\begin{array}{ll}\text { N } 3,88 & 3,73 \%\end{array}$

1-Arseno-4-benzoyl-glutaminsäure. $\left(\mathrm{C}_{12} \mathrm{H}_{12} \mathrm{O}_{5} \mathrm{NAs}\right)_{2}$. Mol.-Gew. 650 .<smiles>O=C(O)CCCCCCCCCCCC(=O)NC(CCC(=O)O)C(=O)NCCCCCC(=O)O</smiles>

bildet ein gelbes Pulver, das etwas in Wasser löslich ist.

$0,2434 \mathrm{~g}$ Substanz gaben bei $16^{\circ}$ und $750 \mathrm{~mm} 8,9 \mathrm{ccm} \mathrm{N}$.

$\begin{array}{cc}\text { Gefunden: } & \text { Berechnet: } \\ \text { i } 4,20 & 4,31 \%\end{array}$

Pentamethylendiamin-dibenzoyl-p-arsinexyd. $\mathrm{C}_{19} \mathrm{H}_{20} \mathrm{O}_{4} \mathrm{~N}_{2} \mathrm{As}_{2}$. Mol.-Gew. 490 .

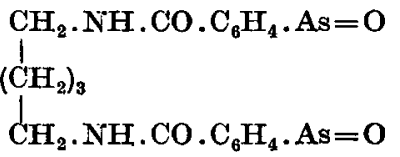

Wenn auch das Pentamethylendiamin oder Cadaverin keine Aminosäure des Eiweißmoleküls ist, so hat es zu letzterem doch ge- 
wisse Beziehungen. Auch ließ die Tatsache, daß das Pentamethylendiamin sich sehr leicht benzoylieren läßt, und daß das wohl charakterisierte Benzoylierungsprodukt geradezu die Isolierungsform dieses Körpers, z. B. aus dem Harn bei gewissen Stoffwechselanomalion ist, ein leicht zu gewinnendes Cadaverinbenzoylarsinoxyd erwarten. Wenn letzteres sich ebenso glatt wie die Benzoylarsinoxyde der Aminosäuren reduzieren ließ, so stand als Reaktionsprodukt ein interessanter, völlig in sich geschlossener, ringförmiger Arsenokörper $\mathbf{z u}$ erhoffen.

Für ausgesprochene Basen ist nouerdings die saure Benzoylierung empfohlen $\left.{ }^{1}\right)$. Bei 30 stündigem Kochen von molekularen Mengen salzsaurem Cadaverin und Dichlorarsinbenzoylchlorid in Benzol am Rückflußkühler fand nur eine sehr geringe Salzsäureentwickelung statt, und es ließen sich die Ausgangsmaterialien fast unverändert wieder zurückgewinnen.

Befriedigend war das Resultat, als der Prozeß bei Gegenwart von viel starkem Alkali vorgenommen wurde.

3,5 g Pentamethylendiamin-chlorhydrat ( $2 / 100$ Mol.) werden in $30 \mathrm{ccm}$ Wasser gelöst, und $10 \mathrm{ccm} 21 / 2-\mathrm{N}$.-Natronlauge hinzugegeben. In zwei Meßzylindern werden $28,5 \mathrm{~g}$ Dichlorarsinbenzoylchlorid $\left({ }^{10} / 100 \mathrm{Mol}.\right)$ und $450 \mathrm{ccm} 21 / 2-N$.-Natronlauge gebracht und von diesen beiden Flüssigkeiten entsprechende Mengen in aoht Portionen der ersten Lösung zugefügt. Vor jedem neuen Zusatz wird das Gefäß in einer Kältemischung gut gekühlt, und dann jedesmal 40-50 Minuten lang auf der Maschine geschüttelt, so daß die ganze Operation etwa 8 Stunden dauert. Nach Beendigung wird die trübe Flüssigkeit durch Asbest filtriert und mit Salzsäure übersättigt. Der hierdurch entstandene Niederschlag wird mit kaltem, dann mit siedendem Wasser ausgewaschen und schließlich mit Alkohol und Aether. Der Rückstand erstarrt an der Luft zu einer weißen, harten, glasigen Masse, die zerrieben ein feines nicht hygroskopisches Pulver darstellt, das in allen Lösungsmitteln unlöslich ist, außer in etwa $5 \%$ iger oder stärkerer Alkalilange. Ausbeute $3,6 \mathrm{~g}$ $=37 \%$ der Theorie.

$$
\begin{aligned}
& 0,1873 \mathrm{~g} \text { Substanz gaben } 0,1160 \mathrm{~g} \mathrm{Mg}_{2} \mathrm{As}_{2} \mathrm{O}_{7} \text {. } \\
& 0,2166 \mathrm{~g} \quad " \quad 0,3685 \mathrm{~g} \mathrm{CO}_{2} \text { und } 0,0818 \mathrm{~g} \mathrm{H} \mathrm{H}_{2} \mathrm{O} \text {. } \\
& \text { Gefunden: Berechnet: } \\
& \text { As } 29,91 \quad 30,61 \% \\
& \text { C } \quad 46,39 \quad 46,53 \% \\
& \text { H } \quad 4,23 \quad 4,08 \%
\end{aligned}
$$

1) H. Franze n, Ber. 42, 2465 (1909). 
Die Erwartungen, durch Oxydation bzw. Reduktion zu analogen Derivaten wie bei den Aminosäuren zu gelangen, erfüllten sich nicht. Die Produkte erschienen nicht einheitlich, und die Analysen ließen keinen bestimmten Sohluß zu.

Leicht und glatt reagiert das Dichlorarsinbenzoylchlorid auch mit höheren Alkoholen.

$$
\begin{gathered}
\text { Arsinoxyd-p-benzoyl-myricylester. } \\
\mathrm{C}_{37} \mathrm{H}_{65} \mathrm{O}_{3} \mathrm{As} \text {. Mol.-Gew. } 632 . \\
\mathrm{C}_{30} \mathrm{H}_{61} \mathrm{O} . \mathrm{CO} . \mathrm{C}_{6} \mathrm{H}_{4} \text {.As }=0 .
\end{gathered}
$$

$\mathrm{Zu}$ einer Lösung von $3 \mathrm{~g}$ Myricylalkohol vom Schmelzpunkt $85,5^{\circ}$ und $1,5 \mathrm{~g}$ Pyridin in $100 \mathrm{ccm}$ Benzol wird bei Stubentemperatur eine Lösung von $2 \mathrm{~g}$ Dichlorarsinbenzoylchlorid in $50 \mathrm{ccm}$ Benzol in kleinen Portionen unter häufigem Umschütteln allmählich hinzugegeben. Nachdem das Reaktionsgemisch einige Stunden sich selbst überlassen ist, wird $1 / 2$ Stunde auf dem Wasserbad unter Rückfluß gelinde erwärmt. Beim Erkalten setzen sich am Grunde des Gefäßes farblose Schmieren an, von denen das Lösungsmittel abgegossen wird, und die wiederholt mit kalter verdünnter Salzsäure ausgesshüttelt werden. Der Rückstand wird dann im Mörser mit Natriumkarbonatlösung durchknetet, wodurch er krümelig wird. Er wird wiederholt mit heißem Wasser ausgewaschen, in Alkohol aufgenommen und die alkoholische Lösung verdunstet. Die Substanz hinterbleibt so in Form weißer Schüppchen.

$$
\begin{array}{ccc}
0,5162 \mathrm{~g} & \text { Substanz gaben } & 0,1305 \mathrm{~g} \mathrm{Mg}_{2} \mathrm{As}_{2} \mathrm{O}_{7} . \\
0,1638 \mathrm{~g} & \text { "refunden: } & 0,4216 \mathrm{~g} \mathrm{CO}_{2} \text { und } 0,1548 \mathrm{~g} \mathrm{H}_{2} \mathrm{O} . \\
\text { Berechnet: } & \text { As } 12,20 & 11,86 \% \\
\text { C } 70,19 & 70,25 \% \\
\text { H } 10,59 & 10,30 \%
\end{array}
$$

Dieses Arsinoxyd löst sich leicht in alkoholischen Flüssigkeiten, besonders bei geringem Erwärmen; in Aether und in Kohlenwasserstoffen ist es viel schwerer löslich, ganz unlöslich in Wasser und Laugen.

Wird der Arsinoxyd-p-benzoyl-myricylester in Aceton gelöst und diese Lösung mit 30\%igem Wasserstoffsuperoxyd versetzt, so trübt sich nach einigem Stehen die Lösung. Sie wird bei gelinder Wärme zur Trockne verdunstet, der Rückstand mit Aceton auf genommen, wozu jetzt aber viel mehr wie zuerst nötig ist, und abermals mit etwas Wasserstoffsuperoxyd versetzt. Der beim Einengen der Lösung sich abscheidende Körper, der 
E. Sieburg: Ester aromatischer Areenverbindungen.

$$
\begin{gathered}
\text { Arsinsäure-p-benzoyl-myricylester } \\
\mathrm{C}_{37} \mathrm{H}_{67} \mathrm{O}_{5} \mathrm{As.} \text { Mol.-Gew. } 666 \\
\mathrm{C}_{30} \mathrm{H}_{61} \mathrm{O} \text {.CO. } \mathrm{C}_{8} \mathrm{H}_{4} \cdot \mathrm{AsO}_{3} \mathrm{H}_{2}
\end{gathered}
$$

bildet winzige zugespitzte Blättchen, die in alkoholischen Flüssigkeiten schwerer löslich sind, als das Arsinoxyd.

$$
\begin{aligned}
& 0,1824 \mathrm{~g} \text { Substanz gaben 0,4484 } \mathrm{g} \mathrm{CO}_{2} \text { und } 0,1668 \mathrm{~g} \mathrm{H}_{2} \mathrm{O} \text {. } \\
& \text { Gefunden: } \\
& \begin{array}{lll}
\text { C } & 67,04 & 66,67 \% \\
\text { H } & 10,25 & 10,06 \%
\end{array}
\end{aligned}
$$

Arseno-p-benzoyl-myricylester.$$
\left(\mathrm{C}_{37} \mathrm{H}_{65} \mathrm{O}_{2} \mathrm{As}\right)_{2} \text {. Mol.-Gew. } 1232 .
$$$$
\mathrm{C}_{30} \mathrm{H}_{61} \mathrm{O} \cdot \mathrm{CO} \cdot \mathrm{C}_{6} \mathrm{H}_{4} \cdot \mathrm{As}=\text { As. } \mathrm{C}_{6} \mathrm{H}_{4} \cdot \mathrm{CO} . \mathrm{OC}_{30} \mathrm{H}_{61} \text {. }
$$

Es wird eine gesättigte Lösung des Arsinoxyds in Aceton bereitet, diese am Rückflußkühler in gelindem Sieden erhalten, und von Zeit zu Zeit messerspitzenweise krystallisierte phosphorige Säure hineingegeben, bis die gelbe Ausscheidung sich nicht mehr vermehrt. Alsdann wird abfiltriert, säurefrei gewaschen und getrocknet.

Der Arseno-p-benzoyl-myricylester bildet ein sich etwas fettig anfühlendes fahlgelbes Pulver, das am leichtesten in starkem Alkohol, aber auch in Aether und Benzol löslich ist.

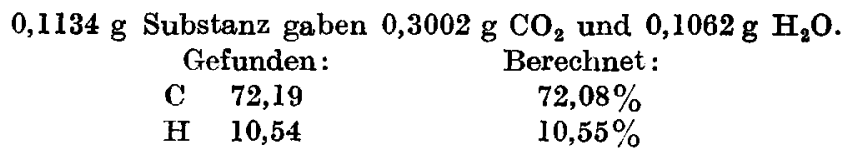

$$
\begin{gathered}
\text { Arsinoxyd-p-benzoyl-cholesterinester. } \\
\mathrm{C}_{34} \mathrm{H}_{48} \mathrm{O}_{3} \text { As. Mol.-Gew. } 579 \text {. } \\
\mathrm{C}_{27} \mathrm{H}_{44} \mathrm{O} \text {.CO. } \mathrm{C}_{6} \mathrm{H}_{4} \text {. As }=0 \text {. }
\end{gathered}
$$

$\mathrm{Er}$ wird dargestellt analog dem Myricylester aus 7,5 $\mathrm{g}$ wasserfreiem Cholesterin und $4 \mathrm{~g}$ Pyridin, und $6 \mathrm{~g}$ Dichlorarsinbenzoylchlorid und $150 \mathrm{ccm}$ Benzol. Das Rohprodukt wird in Aether gelöst, die Lösung etwas eingeengt und mit Benzol versetzt. Es fällt dann das gesuchte Oxyd in weißen Flocken aus. Nach nochmaligem Auflösen in Aether und Fällen mit Benzol ist der Körper rein.

$$
\begin{aligned}
& 0,6224 \mathrm{~g} \text { Substanz gaben } 0,1590 \mathrm{~g} \mathrm{Mg}_{2} \mathrm{As}_{2} \mathrm{O}_{i} \text {. } \\
& 0,1874 \mathrm{~g} \quad, \quad 0,4854 \mathrm{~g} \mathrm{CO}_{2} \text { und } 0,1416 \mathrm{~g} \mathrm{H}_{2} \mathrm{O} \text {. } \\
& \text { Gefunden: Berechnet: } \\
& \text { As } 12,50 \quad 12,95 \% \\
& \text { C } 70,64 \quad 70,46 \% \\
& \begin{array}{l}
\mathrm{H} \quad \mathbf{8 , 3 9} \quad 8,29 \% \\
\hline
\end{array}
\end{aligned}
$$




\section{Arsinsäure-p-benzoyl-cholesterinester \\ $\mathrm{C}_{34} \mathrm{H}_{50} \mathrm{O}_{5}$ As. Mol.-Gew. 613 \\ $\mathrm{C}_{27} \mathrm{H}_{44} \mathrm{O} \cdot \mathrm{CO} \cdot \mathrm{C}_{6} \mathrm{H}_{4} \cdot \mathrm{AsO}_{3} \mathrm{H}_{2}$}

wird durch energische Oxydation des Arsinoxyds in Form feiner spitzer Nädelchen erhalten, die in Alkohol schwerer löslich sind als das Arsinoxyd. Noch schwerer in Alkohol löslich ist das Kaliumsalz, das nach Zusatz alkoholischer Kalilauge zu einer gesättigten alkoholischen Lösung des Arsinsäure-p-henzoyl-cholesterinesters allmählich in gut ausgebildeten dickeren Nadeln ausfällt.

$$
\begin{aligned}
& 0,1736 \mathrm{~g} \text { Substanz gaben } 0,4222 \mathrm{~g} \mathrm{CO}_{2} \text { und } 0,1298 \mathrm{~g} \mathrm{H}_{2} \mathrm{O} \text {. } \\
& \begin{array}{ccc}
\multicolumn{2}{c}{\text { Gefunden: }} & \text { Berechnet: } \\
\text { C } & 66,32 & 66,56 \% \\
\text { H } & 8,18 & 8,52 \%
\end{array}
\end{aligned}
$$

Arseno-p-benzoyl-cholesterinester. $\left(\mathrm{C}_{34} \mathrm{H}_{48} \mathrm{O}_{2} \mathrm{As}\right)_{2}$. Mol-Gew. 1126.

$$
\mathrm{C}_{27} \mathrm{H}_{44} \mathrm{O} \cdot \mathrm{CO} \cdot \mathrm{C}_{6} \mathrm{H}_{4} \cdot \mathrm{As}=\mathrm{As} . \mathrm{C}_{6} \mathrm{H}_{4} \cdot \mathrm{CO} . \mathrm{OC}_{27} \mathrm{H}_{44}
$$

dargestellt durch Reduktion des Arsinoxyds in alkoholischer Lösung mittels phosphoriger Säure, bildet ein feines gelbes Pulver, das in Benzol und Chloroform löslich ist. Die Lösungen in Chloroform sind wenig haltbar, denn beim Stehen an der Luft entfärben sie sich allmählich.

$$
\begin{aligned}
& 0,1518 \mathrm{~g} \text { Substanz gaben } 0,4009 \mathrm{~g} \mathrm{CO}_{2} \text { und } 0,1108 \mathrm{~g}_{2} \mathrm{O} \text {. } \\
& \text { Gefunden: } \\
& \text { C } 71,98 \\
& \mathrm{H} \quad \mathbf{8 , 1 8} \mathbf{8 , 5 2 \%}
\end{aligned}
$$

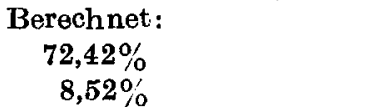

Die Cholesterin-arsenverbindungen geben selbstverständlich die bekannten Cholesterinreaktionen, da bei diesen ja stets kon. zentrierte Schwefelsäure beteiligt ist, die die Ester mehr oder weniger sofort spaltet. Versetzt man dagegen eine alkoholische Lösung, z. B. des Arsinsäure-p-benzoyl-cholesterinesters, mit einer alkoholischen Lösung eines Vertreters aus der Gruppe der Saponinsubstanzen, beispielsweise mit Digitonin, so bleibt das Gemisch klar. Wind a u s'), dem wir die Kenntnis dieser wichtigen Reaktion verdanken, hebt hervor, daß nur das Cholesterin als solches eine Verbindung mit gewissen Saponinen eingeht, Cholesterinester dagegen nicht. Im vorliegenden Falle dient daher das Digitonin als ein bequemes Reagens auf die Reinheit des Körpers, d. h. auf die Abwesenheit. von unverestertem Cholesterin.

1) W ind a us, Ber. 42, 238 (1909). 